\title{
CARACTERIZAÇÃO DE NANOCOMPÓSITOS COM PARTÍCULAS DE CARBETO DE BORO DISPERSAS EM MATRIZ DE POLIETILENO DE ULTRA ALTO PESO MOLECULAR*
}

\section{Resumo}

Flávio James Tommasini ${ }^{1}$ Édio Pereira Lima Junior ${ }^{2}$ Sergio Neves Monteiro ${ }^{3}$ Alaelson Vieira Gomes ${ }^{4}$ Marcelo H Prado da Silva Carla S Cosenza ${ }^{6}$

O estudo teve como objetivo a produção de corpos de prova de nanocompósitos baseados em uma matriz polimérica de polietileno de ultra alto peso molecular (UHMWPE) com carga de carbeto de boro dispersas, nas proporções de 0,0625\%; $0,125 \% ; 0,25 \% ; 0,5 \% ; 1 \%$ e $2 \%$, respectivamente. As respectivas misturas entre os materiais, polímero e carga inorgânica, foram moldadas em prensa hidráulica quente à partir do estado fundido da matriz polimérica. A caracterização dos nanocompósitos com as cargas sem tratamento foi realizada pelas análises de difração de raio-x (DRX) e calorimetria diferencial de varredura (DSC), o que possibilitou a identificação dos planos cristalográficos característicos dos materiais, o grau de cristalinidade da matriz orgânica e suas respectivas temperaturas de fusão. As microestruturas dos materiais, morfologia da carga e adesão interfacial foram observadas através da microscopia eletrônica de varredura (FEGSEM). Através desse estudo foi possível o sugerir o comportamento do polímero com cargas de carbeto de boro dispersas em diversas proporções.

Palavras-chave: Nanocompósitos; Carbeto de Boro, Polietileno de Ultra Alto Peso Molecular, Polímero, Estado Fundido.

\section{CHARACTERIZATION OF NANOCOMPOSITES BASED ON BORON CARBIDE PARTICLES DISPERSE INTO THE ULTRA HIGH MOLECULAR WEIGHT POLYETHYLENE MATRIX}

\section{Abstract}

Study was focused in the production of nanocomposites samples based in polymer matrix of ultra high molecular weight polyethylene (UHMWPE) containing boron carbide fillers in several proportions, 0,0625\%; 0,125\%; 0,5\%; $1 \%$ e $2 \%$, respectively. Heat hydraulic pressing on melting state of the polymeric matrix molded mixtures between the polymer and the inorganic fillers. Characterization of nanocomposites with untreated fillers were performed by $x$-ray diffraction (DRX) and differential scanning calorimetry (DSC), which permitted the identification of crystallinity planes of the materials, crystallinity degrees and respective melt temperatures. The microstructures of the materials, filler morphologies and interface adhesion were observed by scanning electron microscopy (FEGSEM). The research enabled to suggest about polymer behaviour containing several proportions of boron carbide fillers.

Keywords: Nanocomposites, Boron Carbide, Ultra High Molecular Weight Polyethylene, Polymer, Melt State.

1 Desenho Industrial- Projeto de Produto, Post doutorado, Pesquisador, Departamento de Engenharia de Materiais, Instituto Militar de Engenharia, Rio de Janeiro- RJ, Brasil.

2 Engenharia Mecânica, Doutorando em Engenharia Materiais Departamento de Engenharia de Materiais Instituto Militar de Engenharia, Rio de Janeiro- RJ, Brasil.

3 Engenharia de Materiais, Phd, professor, Instituto Militar de Engenharia, Rio de Janeiro-RJ, Brasil. 
4 Engenharia Metalúrgica, Doutor, Professor, Departamento de Engenharia de Mecânica e Materiais, Instituto Militar de Engenharia, Rio de Janeiro-RJ, Brasil.

5 Engenharia Metalúrgica e de Materiais, Doutor, Professor, Departamento de Engenharia de Mecânica e Materiais, Instituto Militar de Engenharia, Rio de Janeiro-RJ, Brasil

6 Engenharia de Materiais, graduando, iniciação científica, Departamento de Engenharia de Mecânica e Materiais, Instituto Militar de Engenharia, Rio de Janeiro-RJ, Brasil.. 


\section{INTRODUÇÃO}

Os materiais poliméricos possuem importantes propriedades e características que os tornam extremamente necessários para os ciclos de produção de diversos produtos presentes no cotidiano das pessoas. Entre os tipos de polímeros que possuem aplicações com finalidades altamente específicas e utilizados para produção de materiais de alto desempenho, existe o polietileno de ultra alto peso molecular. Esse tipo de polímero é conhecido por ser um termoplástico da classe das poliolefinas, cujas cadeias são longas com peso molecular maior do que $1.10^{6} \mathrm{~g} / \mathrm{mol}$ [1-3]. As propriedades e características adquiridas por esse tipo de polietileno são apresentadas através de sua alta resistência mecânica, como impacto, flexão, tração e compressão, assim como, o baixo coeficiente de atrito, alta resistência à abrasão, inércia química e possibilidades diversas para moldagem e processabilidade do material fundido. Todavia, a sua aplicação tem sido limitada pelos baixos módulos de elasticidade e capacidade de carga. Com intuito de melhorar essas características, é usual adicionar cargas que gerem qualidades desejáveis no UHMWPE [4-5]. Kang et al. [6], que observaram acréscimo na dureza, no grau de cristalinidade, na resistência a deformações e na temperatura de fusão ao adicionar micro e nanopartículas de hidroxiapatita no UHMWPE. Em outro estudo Khan e colaboradores [7] reportaram o aumento da resistência mecânica do UHMWPE pela adição de nanotubos de carbono a matriz polimérica. Todavia, Tan et al. [8] estudaram os efeitos de suspensões coloidais de silício em tecidos de UHMWPE e observaram a melhora no desempenho balístico do material, após submissão de impactos com baixa velocidade. A carga analisada na pesquisa foi o carbeto de boro $\left(\mathrm{B}_{4} \mathrm{C}\right)$, devido suas características como dureza, alto módulo de elasticidade e sua alta resistência a fratura [9]. Assim, os compósitos contendo partículas de $\mathrm{B}_{4} \mathrm{C}$ dispersas na matriz de UHMWPE ainda não foram amplamente estudados. Boyhov et al. [10] notaram melhorias nas propriedades mecânicas do UHMWPE quando foi adicionado o carbeto de boro por meios de um moinho de bolas. Similarmente a essa pesquisa, Sharma et al. [11] concluíram que a adição de carbeto de boro ao UHMWPE resultou em uma dureza e resistência a desgaste mais elevados.

O estudo e caracterização dos nanocompósitos de UHMWPE contendo nanopartículas de carbeto de boro dispersas possui alto potencial científico para produção de novos materiais com alto desempenho para utilização em propriedades antibalísticas.

\section{MATERIAIS E MÉTODOS}

O UHMWPE (UTEC 6540 - 8•106 $\mathrm{g} / \mathrm{mol}$ ) foi fornecido pela Braskem, enquanto o carbeto de boro $\left(\mathrm{B}_{4} \mathrm{C}\right)$, cujo tamanho de partícula é menor do que 50 nanômetros, foi adquirido da SkySpring Nanomaterials, Inc. O polímero sob a forma de pó foi submetido a moagem e prévia mistura com as cargas, nas proporções de $0,0625 \%$, $0,125 \%, 0,25 \%, 0,5 \%, 1 \%$ e $2 \%$, a partir de um moinho de bolas, Marconi, modelo MA500, pelo período de 15 horas, sob 200 rpm, com esferas de alumina, na proporção em massa de 6:5 (mistura de:esferas). As misturas foram processadas na prensa hidráulica com aquecimento Solab, modelo SL-11, sob a temperatura de $230^{\circ} \mathrm{C}$, durante 30 minutos. As amostras foram identificadas por: 0, 1, 2, 3, 4, 5 e 6, sendo a amostra 0 referente ao UHMWPE e em proporções crescentes, respectivamente. As análises de difração de raio X (XRD - X-Ray Diffraction) foram realizadas em um 
difratômetro, modelo X'Pert PRO, fabricado pela PANalytical, empregando radiação Ka do Co $(\lambda=0,1789 \mathrm{~nm})$ na faixa $2 \theta$ de 5 a $40^{\circ}$.

As análises por calorimetria diferencial de varredura (DSC - Differential Scanning Calorimetry) foram realizadas utilizando o DSC modelo 404 F1 Pegasus, fabricado pela Netzsch, sob atmosfera inerte de nitrogênio $\left(\mathrm{N}_{2}\right)(70 \mathrm{ml} / \mathrm{min})$, com cadinhos de Alumínio, na faixa de temperatura de 30 a $200^{\circ} \mathrm{C}$. A morfologia, dispersão e interface foram observadas pela análise de microscopia eletrônica de varredura, cujo equipamento foi um FEGSEM (Field Emission Gun - Scanning Electron Microscopy), modelo QUANTA FEG 250, fabricado pela FEI. O material sofreu recobrimento de platina e a energia de tensão de aceleração foi de $20 \mathrm{kV}$. A morfologia foi observada em amostras fraturadas após criogenia.

\section{RESULTADOS E DISCUSSÃO}

A análise de difração de raio-x foi realizada para todas amostras para verificação dos padrões de cristalinidade das amostras puras e processadas, e assim possibilitar a observação de possíveis deslocamentos nos picos cristalinos das amostras poliméricas para detecção da ocorrência de esfoliação ou intercalação nas cargas. Os resultados das análises de XRD foram normalizadas pela maior contagem obtida, referente ao polímero puro. A partir dos espectros foi constatado o comportamento similar para as amostras. Os ângulos de Bragg obtidos estão de acordo com a norma JCPDS 00-054-1982 referente ao polietileno [12]. Os resultados de XRD foram consolidados na Tabela 1, a qual o tamanho do cristalito foi calculado de acordo com a equação de Scherrer modificada [13]. O aumento do tamanho do cristalito calculado foi verificado, provavelmente devido a adição de cargas quando comparado ao UHMWPE, o que pode ser evidencia de alterações nas características cristalográficas na matriz polimérica. (Tabela 1). Através dos resultados preliminares obtidos não é possível sugerir nenhum efeito de intercalação e esfoliação de cargas pelo polímero, tanto nos materiais moídos ou processados sob estado fundido.

Tabela 1. Padrões de difração no ângulo $2 \theta$ de cada amostra dos nanocompósitos

\begin{tabular}{ccccc}
\hline Amostra & $\mathbf{2 \theta}$ & $\mathbf{2 \theta}$ & $\mathbf{2 \theta}$ & $\mathbf{L}(\mathbf{n m})$ \\
\hline 0 & 25,14 & 27,95 & 35,16 & 21,14 \\
\hline 1 & 25,16 & 27,96 & 35,14 & 27,38 \\
\hline 2 & 25,12 & 27,93 & 35,10 & 23,11 \\
\hline 3 & 25,19 & 27,98 & 35,17 & 30,04 \\
\hline 4 & 25,21 & 27,99 & 35,17 & 30,54 \\
\hline 5 & 25,19 & 28 & 35,20 & 36,43 \\
\hline 6 & 25,14 & 27,93 & 35,12 & 27,38 \\
\hline
\end{tabular}

A análise de DSC foi realizada para verificar a influência que a adição de carga à matriz fundida, quanto a temperatura de fusão e cristalinidade da matriz polimérica dos nanocompósitos. Em relação as análises de DSC do primeiro aquecimento dos nanocompósitos (NC) e das misturas moídas foi observada uma uniformidade de comportamento. A Tabela 2 mostra os resultados das análises de DSC. A proximidade das temperaturas de início, máximo e termino de fusão ( $T_{\text {onset, }} \mathrm{T}_{\max } \mathrm{e} \mathrm{T}_{\text {end, }}$, respectivamente) das amostras moídas com carga em relação ao UHMWPE em pó foi observada, entretanto foi observado que as amostras processadas desenvolveram um pequeno decréscimo nos valores de temperatura. A tendência para redução no grau de cristalinidade foi correspondente ao aumento de centros de nucleação pela adição de maior teor de cargas. 
Tabela 2. Resultados das análises de DSC para a temperatura inicial de fusão ( $\left.T_{\text {onset }}\right)$, máximo ponto de fusão ( $\left.T_{\max }\right)$ e temperatura final de fusão ( $\left.T_{\text {end }}\right)$ das amostras de nanocompósitos

\begin{tabular}{ccccccccc}
\hline & \multicolumn{3}{c}{ Moídos } & \multicolumn{4}{c}{ Processados } \\
\hline Amostra & $\mathbf{T}_{\text {onset }}$ & $\mathbf{T}_{\max }$ & $\mathbf{T}_{\text {end }}$ & $\mathbf{X}_{\boldsymbol{c}}$ & $\mathbf{T}_{\text {onset }}$ & $\mathbf{T}_{\max }$ & $\mathbf{T}_{\text {end }}$ & $\mathbf{X}_{\boldsymbol{c}}$ \\
\hline 0 & 132 & 142,5 & 147,5 & 52 & 125 & 135,5 & 140 & 25 \\
\hline 1 & 133 & 142,5 & 147 & 51 & 125 & 136 & 140 & 23 \\
\hline 2 & 132 & 142 & 147 & 52 & 126 & 136 & 141 & 25 \\
\hline 3 & 133 & 142 & 146,5 & 50 & 127 & 135 & 139 & 24 \\
\hline 4 & 133 & 142 & 146 & 50 & 125 & 135 & 140 & 23 \\
\hline 5 & 132,5 & 142,5 & 147 & 54 & 126 & 134 & 139 & 26 \\
\hline 6 & 133 & 142 & 147 & 49 & 125 & 135 & 140 & 22 \\
\hline $\begin{array}{c}\text { Desvio } \\
\text { Padrão }\end{array}$ & 0,35 & 0,18 & 0,38 & 1,71 & 0,77 & 0,72 & 0,78 & 1,32 \\
\hline Média & 133 & 142 & 147 & 51 & 126 & 135 & 140 & 24 \\
\hline
\end{tabular}

A observação da microestrutura dos nanocompósitos processados, as superfícies dos materiais, fraturadas por criogenia foram analisadas por microscopia eletrônica. As imagens obtidas a partir do polímero puro (UHMWPE) possibilitaram a observação característica da superfície da matriz termoplástica com boa fluidez e homogênea (Figura 1a). A respeito ds partículas de $\mathrm{B}_{4} \mathrm{C}$ foi possível observar que estão dispostas em agregados sob dimensões nanométricas, com formas prismáticas alongadas (Figura 1b)

(1a)

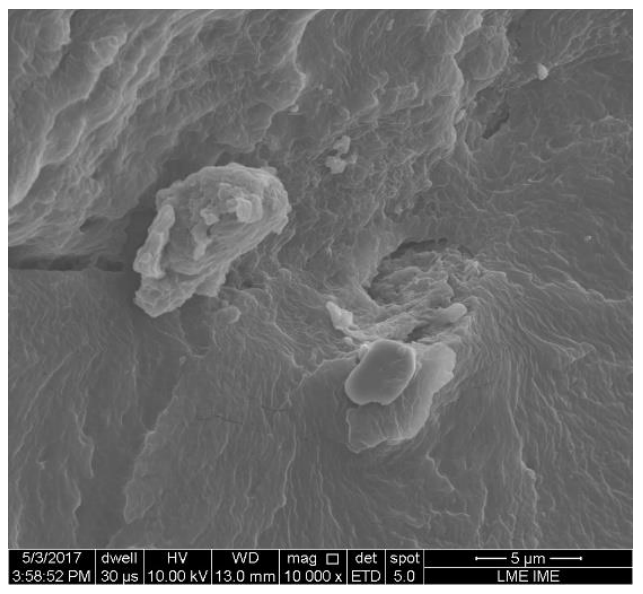

(1b)

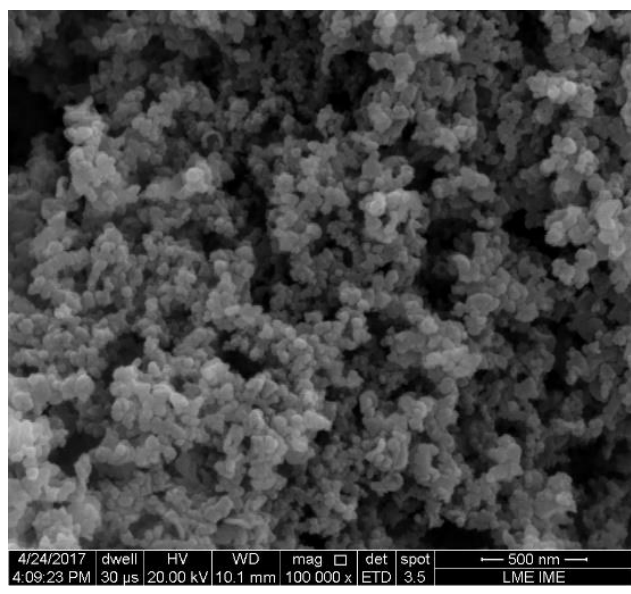

Figura 1a e 1b. Imagens de FEGSEM da matriz polimérica de UHMWPE em 500x \100um (a) e agregados de partículas de carbeto de boro em 100.000x \100nm (b).

A partir da visualização das superfícies dos nanocompósitos $(0,0625 \% ; 0,125 \%$; $0,25 \% ; 0,5 \% ; 1 \%$ e $2 \%$ ), foi possível verificar algumas diferenças entre as determinadas morfologias e microestruturas formadas. Os nanocompósitos 0,0625\% apresentaram uma morfologia da matriz mais fluida e regular, comparada ao material contendo a proporção de carga na matriz em $0,125 \%$ (Figura $2 \mathrm{a}$ e $2 b$ ). Esse efeito pode ser atribuído ao aumento na proporção da carga e consequente maior número de pontos de nucleação dispersos na matriz, o que pode ter diminuído a fluidez do material durante fusão e resfriamento após processamento.

Os nanocompósitos contendo $0,25 \%$ de carga mostrou o mesmo efeito na aparência da matriz, e como esperado, as outras matrizes contendo maior teor de carga dispersa desenvolveram a mesma tendência, e se tornaram com mais quebradiça. Entretanto, 
os nanocompósitos nas maiores proporções, $1 \%$ e $2 \%$, apresentaram em suas superfícies, as partículas de carbeto de boro sem interação interfacial com a matriz polimérica. Esse efeito foi atribuído à falta de interação interfacial entre as fases orgânica e inorgânica, respectivamente (Figura 3a e 3b).
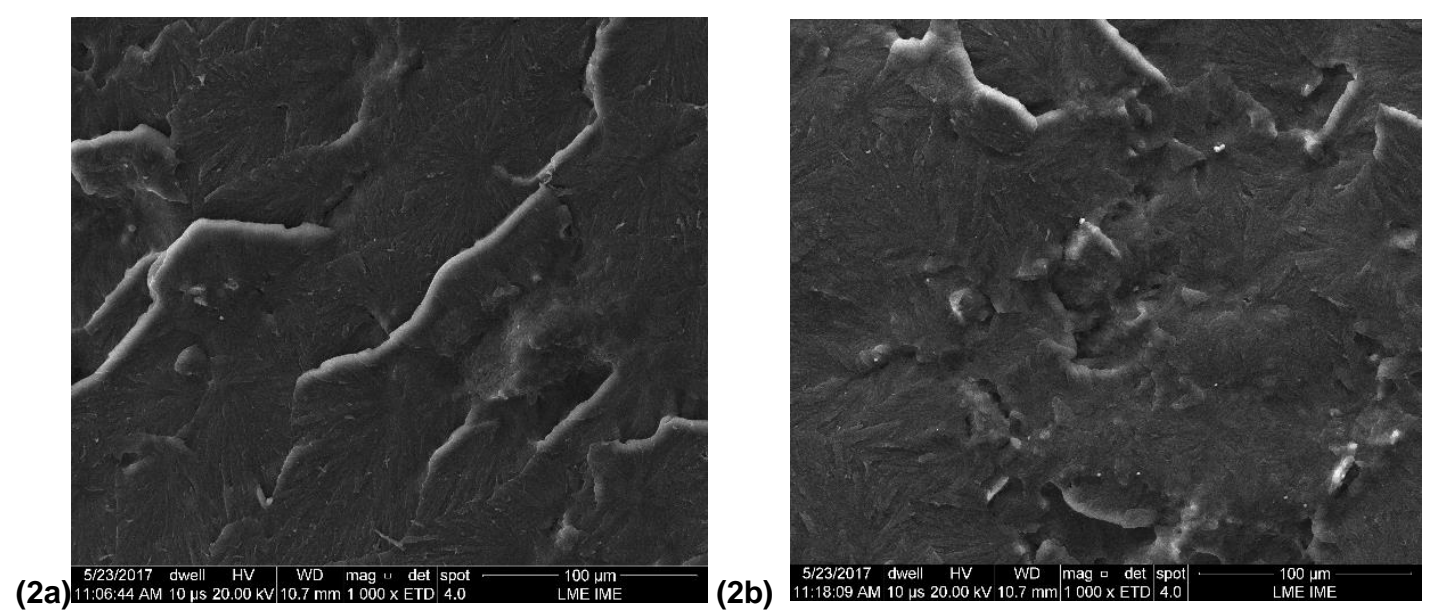

Figura 2a e 2b. Imagens de microscopia eletrônica de varredura (FEGSEM) dos

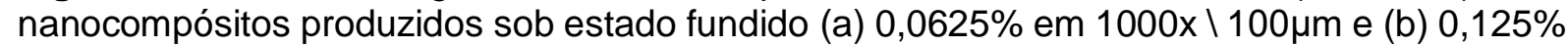

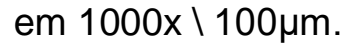

As imagens dos nanocompósitos em magnitudes maiores possibilitou a observação de maior quantidade de pontos de cristalização da matriz polimérica, possivelmente devido a presença da carga $\mathrm{B}_{4} \mathrm{C}$ (Figura 4a).

(3a)

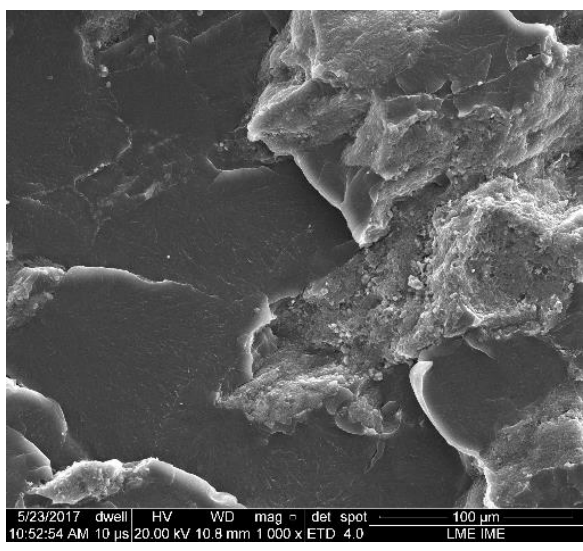

(3b)

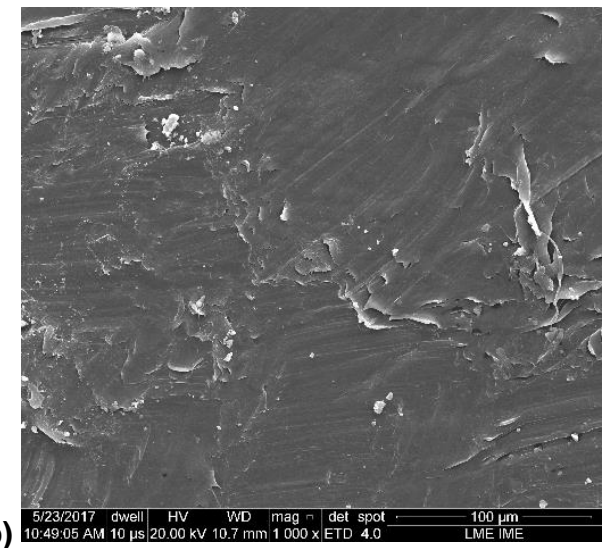

Figura 3a e 3b. Imagens de microscopia de FEGSEM das matrizes das amostras de

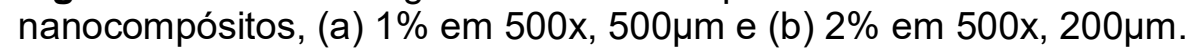

Através da observação da Figura 4b foi possível observar que o processamento do polímero possibilitou o enbebedamento das partículas inorgânicas pela matriz orgânica, mesmo sem possuir afinidade interfacial pelas fases orgânica e inorgânica. 
(4a)
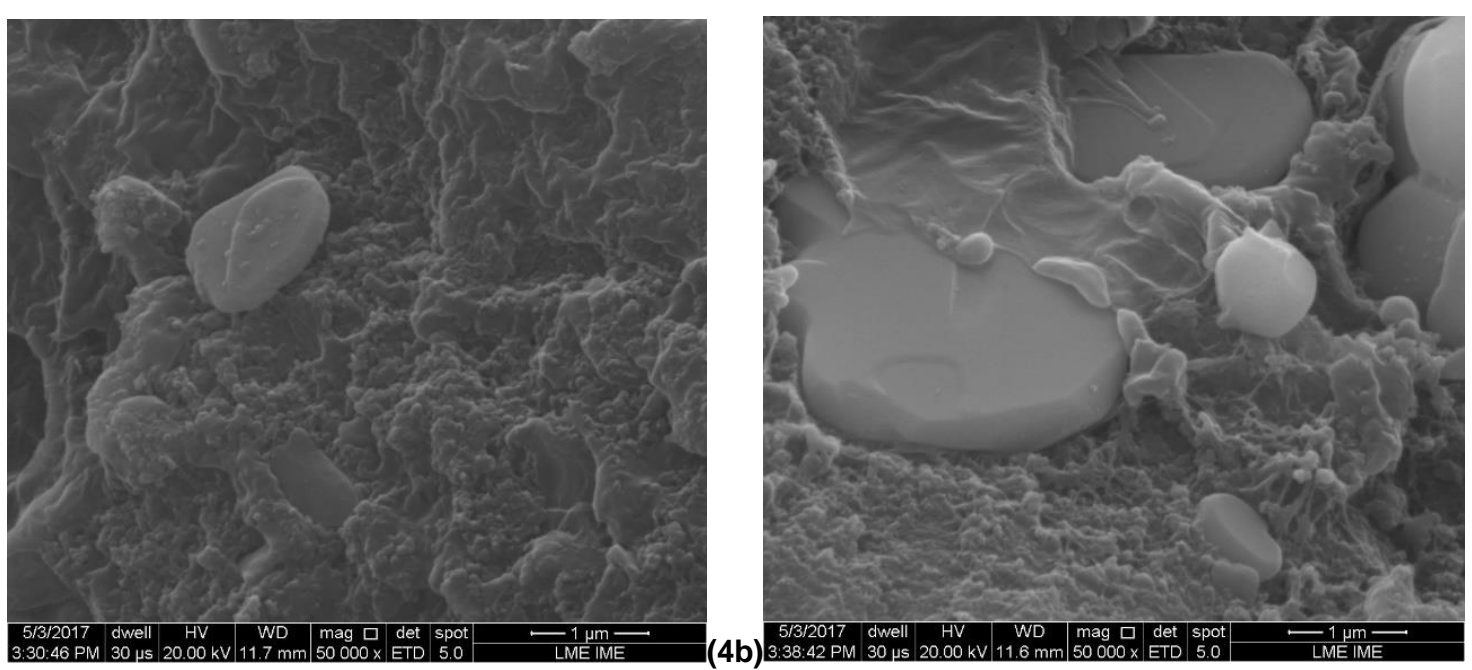

Figura 4a e 4b. Imagens de locais de cristalização da matriz polimérica (a) 1,000\% em $50.000 x, 1 \mu \mathrm{m}$ e partículas de carbeto de boro embebedadas (b) 2,000\% em 50.000x, $1 \mu \mathrm{m}$; respectivamente.

\section{CONCLUSÃO}

A partir dos resultados as análises obtidas, constatou-se que a adição de carbeto de boro alterou algumas propriedades cristalográficas e específicas do arranjo cristalino do polímero, observada através da análise de difração de raio-x (XRD). A redução do grau de cristalinidade por DSC pode ser associada ao aumento do número de centros de nucleação que dificultam a recristalização da matriz polimérica. A análise de microscopia eletrônica de varredura (FEGSEM) possibilitou a observação de certo grau de fluidez das amostras, sendo apresentadas algumas alterações devido às distintas proporções de carga (carbeto de boro) nos diferentes NC e a característica lamelar do material inorgânico. O caráter lamelar das cargas e os resultados obtidos pela análise XRD não podem sugerir uma possível esfoliação do material após o processamento, possivelmente pelo processo estático de moldagem. Consequentemente, pela observação realizada por microscopia eletrônica de varredura, foi possível verificar a diferença de fluidez dos diferentes nanocompósitos produzidos, influenciadas pelas distintas proporções de carga, como pode ser visualizada pelas diferenças de cristalinidade observadas na análise de DSC.

\section{Agradecimentos}

Nós agradecemos a Coordenação de Aperfeiçoamento de Pessoal de Nível Superior (CAPES) e ao Instituto Militar de Engenharia (IME) pelo suporte nesse trabalho.

\section{REFERÊNCIAS}

1 D. Xiong and S. Ge. Friction and wear properties of UHMWPE/Al2O3 ceramic under different lubricating conditions. Wear, vol. 250, n. 1, pp. 242-245, 2001.

2 P. Barbour, M. Stone, and J. Fisher, "A study of the wear resistance of three types of clinically applied UHMWPE for total replacement hip prostheses. Biomaterials, vol. 20, n. 22, pp. 2101-2106, 1999.

3 X. Dangsheng. Friction and wear properties of uhmwpe composites reinforced with carbon fiber. Materials letters, vol. 59, no. 2,pp. 175-179, 2005. 
4 Baker, D.A., Hastings, R.S., Pruitt, L. (2000) Compression and Tension Fatigue Resistance of Medical Grade Ultra High Molecular Weight Polyethylene: The Effect of Morphology, Sterilization, Aging and Temperature. Polymer, 41, 795-808.

5 Chanda, A., Mukhopadhyay, A. K., Basu, D., Chatterjee, S. (1997) Wear and Friction Behaviour of UHMWPE-Alumina Combination for Total Hip Replacement. Ceramic International, 23, 437-447.

6 X. Kang, W. Zhang, and C. Yang. Mechanical properties study of micro- and nanohydroxyapatite reinforced ultrahigh molecular weight polyethylene composites. Journal of Applied Polymer Science, vol. 133, no. 3, 2016.

7 M. R. Khan, H. Mahfuz, A. Adnan, I. Shabib, and T. Leventouri. Elastic properties of UHMWPE-SWCNT nanocomposites fiber: An experimental, theoretic, and molecular dynamics evaluation. Journal of materials engineering and performance, vol. 22, no. 6,pp. 1593-1600, 2013.

8 V. Tan, T. Tay, and W. Teo. Strengthening fabric armour with silica colloidal suspensions. International journal of solids and structures, vol. 42, no. 5, pp. 15611576, 2005.

9 K. Sairam, J. Sonber, T. C. Murthy, C. Subramanian, R. Hubli, A. Suri. Development of B4C-HfB2 composites by reaction hot pressing. International Journal of Refractory Metals and Hard Materials, vol. 35, pp. 32-40, 2012.

10 A. Boykov, V. V. Tcherdyntsev, and V. Gulbin. Structure and mechanical properties of ultrahigh molecular weight polyethylene filled with tungsten, carbon black, and boron carbide or nitride. Composites: Mechanics, Computations, Applications: An International Journal, vol. 7, no. 4, 2016.

11 S. Sharma, J. Bijwe, and S. Panier. Assessment of potential of nano and micro-sized boron carbide particles to enhance the abrasive wear resistance of UHMWPE.

Composites Part B: Engineering, vol. 99, pp. 312-320, 2016.

12 Russel, K, B., Heyding, R., Polymer, vol. 38, pp. $1409,1997$.

13 A. Monshi, M. R. Foroughi, M. R. Monshi. Modified Scherrer Equation to Estimate More Accurately Nano-Crytsallite Size Using XRD. World Journal of Nano Science and Engineering, 2, 154-160, 2012. 